\title{
Podometría, práctica deportiva e índice de masa corporal asociada al dolor podal en niños de 12 a 14 años de escuelas de formación deportiva de fútbol en la localidad de Sincelejo
}

\author{
Kelly Díaz Theran ${ }^{1}$ \\ Fecha: Recibido octubre 30 de 2014 - Aceptado diciembre 11 de 2014
}

\begin{abstract}
Resumen
En muchas ocasiones los niños que realizan práctica deportiva, especialmente fútbol, pueden sentir dolor en los pies en algún momento (antes, durante o después de la práctica), debido a diferentes agentes que pueden estar asociados a factores extrínsecos de la práctica deportiva como el calzado, el tipo de terreno, el mal uso de vendajes o también por factores intrínsecos como aumento en el índice de masa corporal (IMC) o tipología del pie. El objetivo es establecer la asociación del dolor podal con variables podométricas, de la práctica deportiva e Índice de masa corporal (IMC) en niños de 12 a 14 años de escuelas de formación deportiva de fútbol de la ciudad de Sincelejo. La metodología usada es un enfoque empírico-analítico, un estudio correlacional de corte transversal. Se realizó un muestreo probabilístico simple. Se evaluaron 233 niños de la ciudad de Sincelejo. Se utilizó el "método Herzco" para variables podométricas; el cálculo del IMC se realizó según los patrones de crecimiento de la OMS.
\end{abstract}

Palabras clave: dolor, pie, medidas, fútbol, niño.

\section{Pedometry, sports and body mass index associated with foot pain in children 12-14 years of sports training school football in the town of Sincelejo}

\begin{abstract}
Many times children doing sports, especially football, can feel foot pain at some point (before, during or after practice) because different agents may be associated with extrinsic factors of sport as footwear, type of terrain, the misuse of bandages or also by intrinsic factors such as increased body mass index (BMI) or foot type. Objective is to establish the association between foot pain podométricas variables of sport and body mass index (BMI) in children 12-14 years of sports training schools football Sincelejo. Methodology used is an empirical-analytical approach, a correlational cross-sectional study. 233 children from the city of Sincelejo were evaluated. "Herzco Method" podométricas the variables used; IMC calculation was performed according to the growth patterns of OMS.
\end{abstract}

Keywords: pain, foot steps, football, kid

${ }^{1}$ Fisioterapeuta y Coordinadora académica del Programa de Ciencias del Deporte de la Corporación Universitaria del Caribe CECAR. kelly.diaz@cecar.edu.co 
Kelly Díaz Theran - Podometría, práctica deportiva e índice de masa corporal asociada al dolor podal en niños

\section{Introducción}

El presente artículo establece la asociación del dolor podal con variables podométricas de la práctica deportiva e IMC en niños de 12 a 14 años de las escuelas de formación deportiva de fútbol de la ciudad de Sincelejo.

Según autores como Barton, Levinger, Crossley, Webster y Menz (2011), en muchas ocasiones los niños que realizan práctica deportiva, especialmente fútbol, pueden sentir dolor en los pies en algún momento; antes, durante o después de la práctica, debido a diferentes agentes que pueden estar asociados a factores extrínsecos de la práctica deportiva como el calzado, el tipo de terreno, el mal uso de vendajes o también por factores intrínsecos, como aumento en el índice de masa corporal (IMC) o tipología del pie.

Diversos estudios evidencian tanto variaciones en las características de la morfología del pie, como asociados a la presencia del dolor, factores intrínsecos como pronación excesiva del mismo en el despegue, lo que provoca tendinitis del compartimento profundo del pie, por ejemplo, su disposición morfológica (Barton et al., 2011). Otros autores relacionan las causas como factores extrínsecos en virtud de la edad (Arizmendi, Pastrana \& Rodríguez, 2004), el género (Hernández, 2006), la raza y la actividad que cumple el individuo (Gómez, 2010). Sin embargo, en Colombia poco se ha publicado sobre las particularidades de esta extremidad en nuestra población general, hecho que motiva el desarrollo de estudios epidemiológicos que caractericen la morfología del pie en niños que practiquen fútbol.

Uno de los métodos usados para la evaluación y diagnóstico del pie, consiste en el análisis de la huella plantar a través del "método Herzco" de Hernández (1999), la cual es ampliamente utilizada en el ámbito deportivo. A través de ella, permite ver la relación porcentual (\%) de la anchura metatarsiana y la anchura de la bóveda plantar y obtener una clasificación del pie en normal, plano, cavo y algunas combinaciones entre estos tipos.
Esta investigación tributa en conocimiento y beneficio, no solo de la comunidad académica, sino a los padres de familia e instituciones deportivas, la posibilidad de conocer la causa del dolor podal en los niños futbolistas, brindando la probabilidad de realizar correcciones en la alineación postural por medio de plantillas ortopédicas, taloneras o vendajes e incrementar la práctica de la flexibilidad, fortalecimiento muscular con el fin de mejorar o potenciar el rendimiento deportivo.

De igual manera, según plantean Cantó, García, Cantó y Padrós (2007), los datos de esta investigación son un aporte para la industria del calzado deportivo, al ofrecer datos antropométricos importantes para un diseño de calzado más ergonómico, apropiado y de acuerdo con el terreno para la práctica deportiva del fútbol en niños.

Los resultados son un aporte a las ciencias de la salud y el deporte, en especial en el área de la actividad física y el entrenamiento deportivo, convirtiéndose en un referente para la evaluación de variables antropométricas, podométricas y de la práctica deportiva que puedan estar asociadas al dolor podal; propende a la detección temprana de factores desencadenantes del dolor que muchas veces ocasiona que los niños tengan que retirarse de la práctica deportiva. Servirá de insumo para implementar estrategias de prevención de lesiones deportivas y al mejoramiento de la práctica deportiva.

\section{Metodología}

El objetivo general de este trabajo de investigación es establecer la asociación del dolor podal con variables podométricas, de la práctica deportiva e índice de masa corporal en niños de 12 a 14 años de escuelas de formación deportiva de fútbol de la ciudad de Sincelejo, para lo cual iniciaremos determinando el índice de masa corporal de los niños, seguidos de las dimensiones podométricas basadas en el "método Herzco", las variables de la práctica deportiva y su incidencia y las características del dolor podal en los niños mencionados. 
BÚSQUEDA - Julio / Diciembre de 2014 - No. 13 (60 - 67)

El presente trabajo de investigación, bajo el enfoque empírico-analítico es un estudio correlacional de corte transversal; se realizó un muestreo probabilístico simple, mediante el procedimiento de selección por números aleatorios. Cada marco muestral en Excel se ordenó por escuela deportiva y luego los participantes se ordenaron por orden alfabético.

Se inició la recolección de información por medio del consentimiento informado; se notificó y pidió permiso a los padres de familia o acudientes, quienes aceptaron y firmaron la participación de su hijo en el estudio, además, se utilizó un instrumento registro de datos sociodemográficos, de la práctica deportiva e índice de masa corporal, al igual que una encuesta de caracterización del dolor en el último año.

\section{Procedimiento}

Los pasos que se han seguido en este proceso han sido:

- Aleatorización y reclutamiento de los niños.

- Aceptación y firma del consentimiento informado por parte de entrenadores.

- Evaluación de variables sociodemográficas, antropométricas y de la práctica deportiva.

- Evaluación del dolor podal en el último año.

- Toma de la huella plantar.

- Aplicación del "método Herzco".

- Sistematización, tabulación y graficación.

- Análisis de información, discusión de resultados y realización del informe final.

El tiempo promedio de aplicación de los instrumentos por cada niño fue:

- Evaluación de variables sociodemográficas, antropométricas y de la práctica deportiva: 10 minutos.

- Evaluación del dolor podal: 5 minutos.

- Toma de la huella plantar: 5 minutos por cada evaluador.

\section{Resultados}

\section{Variables sociodemográficas}

Se evaluaron 233 niños (sexo masculino) de 12 a 14 años de 9 escuelas de formación deportiva de fútbol de la ciudad de Sincelejo, con un promedio de 7 años de escolaridad (tabla 1).

Tabla 1. Descriptivos de variables cuantitativas

\begin{tabular}{|l|r|r|r|r|r|}
\hline \multicolumn{5}{|c|}{ Estadísticos descriptivos } \\
\hline & \multicolumn{1}{|c|}{ N } & Mínimo & Máximo & \multicolumn{1}{c|}{ Media } & Desv. típ. \\
\hline Edad (meses) & 233 & 12 & 14 & 12,98 &, 737 \\
\hline Nivel de escolaridad (años) & 233 & 3 & 9 & 7,24 & 1,207 \\
\hline Antigüedad en la escuela de fútbol & 233 & 1 & 72 & 23,39 & 18,449 \\
(meses) & & & & & \\
\hline Frecuencia de entrenamiento & 233 & 1 & 5 & 2,84 &, 713 \\
semanal (días) & & & & & 1,255 \\
\hline Talla (cm) & 233 & 123 & 177 & 153,07 & 9,071 \\
\hline Peso (kg) & 233 & 23 & 76 & 44,96 & 2,62004 \\
\hline Índice de Masa Corporal(kg/m2) & 233 & 9,53 & 27,57 & 19,0820 & 11,66645 \\
\hline Medida podometrica derecha (cm) & 233 & 11,58 & 84,52 & 55,9674 & 11,57295 \\
\hline Medida podometrica Izquierda (cm) & 233 & 14,29 & 94,62 & 57,8478 & 9,701 \\
\hline Duración del dolor podal (días) & 233 & 0 & 90 & 2,00 & \\
\hline N válido (según lista) & 233 & & & & \\
\hline
\end{tabular}

Fuente: Datos obtenidos del análisis en SPSS 
Kelly Díaz Theran - Podometría, práctica deportiva e índice de masa corporal asociada al dolor podal en niños

\section{Variables podométricas e IMC}

Los niños contaban con un índice de masa corporal promedio de 19,08 kg/m2, el 80,68\% clasificado como normal; el $8,6 \%$ con bajo peso y el $10,72 \%$ con sobrepeso y obeso. La tipología de pie derecho predominante, medida con el "método Herzco" fue el pie cavo, seguido del pie normal cavo y con menor prevalencia el pie plano. Por su parte, la tipología de pie izquierdo predominante fue el pie cavo, seguido del pie normal cavo y con menor prevalencia el pie plano normal (tabla 2).

Tabla 2. Descriptivos de variables cualitativas

\begin{tabular}{|c|c|c|c|}
\hline Categoría & Variable & Muestra $(\mathrm{n}=\mathrm{xxx})$ & Frecuencia \\
\hline \multirow[t]{22}{*}{ Práctica Deportiva } & Posición de juego & & \\
\hline & Arquero & $8.6 \%$ & 20 \\
\hline & Defensa & $30.5 \%$ & 71 \\
\hline & Volante & $41.2 \%$ & 96 \\
\hline & Delantero & $19.7 \%$ & 46 \\
\hline & Tipo de calzado de práctica deportiva & & \\
\hline & Tenis & $19.3 \%$ & 45 \\
\hline & Tenis guayo & $6.0 \%$ & 14 \\
\hline & Guayo tache redondo de goma & $56.2 \%$ & 131 \\
\hline & Guayo de tache rectangular de pasta & $18.0 \%$ & 42 \\
\hline & Guayo de tache metálico & $0.4 \%$ & 1 \\
\hline & Calzado adecuado para la práctica deportiva & & \\
\hline & Si & $63.1 \%$ & 147 \\
\hline & No & $36.9 \%$ & 86 \\
\hline & Terreno de juego & & \\
\hline & Sintética & $0 \%$ & 0 \\
\hline & Cancha de arena y/o tierra & $99,1 \%$ & 231 \\
\hline & Grama & $0.9 \%$ & 2 \\
\hline & Cemento & $0 \%$ & 0 \\
\hline & Uso de plantillas & $6,0 \%$ & 14 \\
\hline & Uso de taloneras & $3,0 \%$ & 7 \\
\hline & Uso de vendaje en el tobillo & $5,6 \%$ & 13 \\
\hline \multirow[t]{10}{*}{ Podométricas e IMC } & Índice de Masa Corporal & & \\
\hline & Bajo peso & $8.6 \%$ & 20 \\
\hline & Normal & $80.7 \%$ & 22 \\
\hline & Sobrepeso & $9.4 \%$ & 22 \\
\hline & Obeso & $1.3 \%$ & 3 \\
\hline & Tipología del pie derecho (método Herzco) & & \\
\hline & Pie plano & $5.6 \%$ & 13 \\
\hline & Pie plano normal & $2.1 \%$ & 5 \\
\hline & Pie normal & $26.6 \%$ & 62 \\
\hline & Normal cavo & $26.6 \%$ & 62 \\
\hline
\end{tabular}

Fuente: Datos obtenidos del análisis en SPSS 
BÚSQUEDA - Julio / Diciembre de 2014 - No. 13 (60 - 67)

\begin{tabular}{|c|c|c|c|}
\hline & Cavo & $36.5 \%$ & 85 \\
\hline & Cavo fuerte & $2.1 \%$ & 5 \\
\hline & Cavo extremo & $0.4 \%$ & 1 \\
\hline & Tipología del pie izquierdo (método Herzco) & & \\
\hline & Pie plano & $5.6 \%$ & 13 \\
\hline & Pie plano normal & $1.7 \%$ & 4 \\
\hline & Pie normal & $22.3 \%$ & 52 \\
\hline & Normal cavo & $26.6 \%$ & 62 \\
\hline & Cavo & $39.1 \%$ & 91 \\
\hline & Cavo fuerte & $3.0 \%$ & 7 \\
\hline & Cavo extremo & $1.7 \%$ & 4 \\
\hline \multirow[t]{19}{*}{ Dolor podal } & Dolor podal en el último año & $15,9 \%$ & 37 \\
\hline & Causa & & \\
\hline & Traumática & $7.3 \%$ & 17 \\
\hline & Sobreuso & $5.6 \%$ & 13 \\
\hline & Otra & $3.0 \%$ & 7 \\
\hline & Manifestación del dolor según la práctica deportiva & & \\
\hline & Antes & $1.3 \%$ & 3 \\
\hline & Durante & $3.0 \%$ & 7 \\
\hline & Después & $10.3 \%$ & 24 \\
\hline & Localización del dolor podal & & \\
\hline & Antepié & $3.0 \%$ & 7 \\
\hline & Mediopié & $3.4 \%$ & 8 \\
\hline & Retropié & $6.4 \%$ & 15 \\
\hline & Dorso del pié & $1.3 \%$ & 3 \\
\hline & Planta del pié & $1.7 \%$ & 4 \\
\hline & Lateralidad del dolor podal & & \\
\hline & Derecha & $7.7 \%$ & 18 \\
\hline & Izquierda & $3.0 \%$ & 7 \\
\hline & Bilateral & $5.2 \%$ & 12 \\
\hline
\end{tabular}

\section{Variables de la práctica deportiva}

La antigüedad promedio en la escuela deportiva fue de 23 meses, con una frecuencia de entrenamiento semanal promedio de 2 días. La posición de juego más común en los niños fue volante, seguida de defensa. El terreno de juego más empleado para la práctica de fútbol fue cancha de arena, seguida de cancha de grama. El tipo de calzado más utilizado por los niños es el guayo tache redondo, seguido del tenis. El $6 \%$ usa regularmente plantillas, el 3\% taloneras y el $5.6 \%$ vendajes en el tobillo (tabla 2 ).

\section{Variables asociadas al dolor podal}

Se encontró una prevalencia del dolor podal en el último año de $15.9 \%$ (tabla 2) con una duración promedio de 2 días (tabla 1). La causa más común fue traumática, presentándose el dolor principalmente después (antes, durante, después) de la práctica deportiva. La localización predominante fue el retropié, y principalmente en el pie derecho (derecho, izquierdo, bilateral) (tabla 2). 
Kelly Díaz Theran - Podometría, práctica deportiva e índice de masa corporal asociada al dolor podal en niños

\section{Discusiones}

El estudio se realizó en la ciudad de Sincelejo, con una muestra total de 233 niños entre 12 y 14 años, de las escuelas de formación deportiva en fútbol afiliadas a la Asociación de Escuelas de Fútbol de Sucre, ASOSUCRE, resultados que se asemejan a la investigación en donde la población fue de 398, tal como lo plantearon Dvorak, Junge, Chomiak y Graf (2000). En otra investigación a cargo de Gómez, Franco, Nathy, Valencia, Vargas y Jiménez (2009) en la ciudad de Cali, realizada a mujeres deportistas, la población en estudio fue de 106, cifras por debajo de este estudio, y en la misma ciudad, con 77 mujeres sedentarias estudiantes de la Escuela Nacional del Deporte. De igual manera, en otro estudio, la muestra consistió en 118 niños y adolescentes en edades 8-18 años. Asimismo, en Curitiba los autores Wilson, Samuelson y Palermo (2010), realizaron un estudio donde la muestra fue 310 diferentes atletas por encima de los resultados de esta investigación.

En lo relacionado con las variables de estudio en la presente investigación, los niños mostraron un índice de masa corporal promedio de 19,08 k/ m2; clasificado como normal, el 80,68\%; con bajo peso, el $8,6 \%$, y con sobrepeso y obeso el $10,72 \%$. Al revisar un estudio, similar el IMC es 24.02, con 3 niños (2,5\%) están clasificados como bajo peso, 70 niños $(59,3 \%)$ fueron clasificados como de peso saludable, 12 niños (10,0 \%) siendo clasificados con sobrepeso, y 33 niños (28,0\%) fueron clasificados como obesos, datos que no muestran diferencias significanticas en cuanto al IMC al estar los dos datos en la clasificación normal de acuerdo con la Organización Mundial de la Salud, el actual estudio muestra una clasificación de normal mayor que el de Oregón, realizado por Wilson (2010).

Continuando con la discusión en lo relacionado con la tipología del pie derecho, el predominante fue el pie cavo, seguido del pie normal y con menor prevalencia el pie plano; por otra parte, la tipología de pie izquierdo predominante fue el pie cavo, seguido del pie normal y con menor prevalencia el pie plano, datos que difieren con la investigación denominada Alteraciones de la huella plantar en función de la actividad física que se realizó en tres grupos de mujeres con distintos niveles y tipo de actividad física: (sedentarias, jugadoras de élite de fútbol sala y hockey hierba); con respecto al grupo evaluado de futbol sala, se han encontrado diferencias significativas en el pie derecho tras el periodo de intervención, no hallándose estas diferencias en el pie izquierdo. Las diferencias detectadas mostraban una dirección hacia el aplanamiento del pie tras el periodo de intervención (Berdejo, Lara, Martínez, Cachón \& Lara, 2013). Estos resultados pueden deberse a la distinta función que realizan los pies en esta modalidad deportiva. Autores como Aydog, Özçakar, Tetik, Demirel y Doral (2005) obtuvieron resultados similares en futbolistas. Estos autores justificaron este hecho explicando el uso de pie dominante en fútbol; el pie que más funciones realiza (el dominante) verá modificada su morfología por diferentes tensiones musculares y ligamentosas y acabará dando valores más altos en los diferentes métodos de medición. Estos autores, también proponen que el hecho de ser deportista de alto nivel y estar sometido a un entrenamiento intenso, puede provocar también estos cambios estructurales en el sistema músculo esquelético y ligamentoso. Además, según Hamill, Bates, Knutzen y Kirpatrick (1989), otra de las causas a las que se puede deber esta diferencia entre pies, es a la actividad muscular del pie plano que se verá incrementada. Así, el pie que tiene más actividad o función, en este caso el pie derecho, mostraría una tendencia a aplanarse. Por lo tanto, además se podría considerar que el gesto técnico de pisar el balón y el de parar el balón con la zona del antepié plantar, gestos muy utilizados en fútbol sala, provocan un desplazamiento hacia arriba de la cabeza de los metatarsianos y consecuentemente un estiramiento de la fascia plantar, que puede dar una tendencia a pie plano, dato que difiere de esta investigación debido a que el pie plano normal obtuvo un $1.7 \%$ en menor prevalencia.

Esto se complementa con la teoría de Sirgo y Aguado (1991) que demostraron las adaptaciones agudas que se producían en el pie como consecuencia de una situación de competición 
BÚSQUEDA - Julio / Diciembre de 2014 - №. 13 (60 - 67)

deportiva (un partido de voleibol); estos autores observaron un aumento de la huella tanto longitudinalmente como transversal, existiendo variaciones en función de la composición corporal (entre ectomorfos y mesomorfos). Según estos autores, es de esperar que estas modificaciones agudas se hagan crónicas en el tiempo en una persona deportista que somete a sus pies a una gran carga y esfuerzo. Además se ha encontrado un aumento del porcentaje de la huella plantar mediante el "método Herzco". Dato que se asemeja a los hallados por el investigador López et al. donde encontraron el mismo tipo de pie en futbolistas de distintas categorías; los resultados indicaban que la mayoría de los sujetos presentaban un pie normal y más valgo en el ángulo tibio calcaneo del pie derecho que del izquierdo. En cambio, al utilizar el "método Herzco" los resultados de morfotipo de pie se aproximan más hacia el cavo. Además, López, Meana, Vera y García (2006) encontraron un pie cavo asociado a un valgo de calcáneo, de manera similar a lo que se ha encontrado en esta investigación, en la que la tendencia habitual es hacia una dilatación de la huella en todas sus partes (antepié, mediopié y retropié), aunque según la disciplina y las actividades deportivas, se verán unas zonas más afectadas que otras. Es así que Elvira, Vera, Meana y García (2008) encontraron que la marcha atlética no provocaba asimetría ni adaptaciones concretas hacia un tipo de pie. No obstante, obtuvieron diferencias significativas entre pies. Dato que difiere de esta investigación pues se encontró en mayor prevalencia el pie cavo y no se halló significancia estadística entre la tipología del pie y el dolor podal.

Otra investigación que guarda relación con el presente estudio, por la prevalencia del pie cavo y con la cual se puede contrastar, es la denominada "la prevalencia de pie plano en niños y niñas en las edades de 9 a 12 años", realizado por Hernández (2006) cuyos resultados exponen que los alumnos de $6^{\circ}$ año, arrojaron la mayor cantidad de pie plano, en particular los hombres; las mujeres de $5^{\circ}$ grado dieron la mayor cantidad de pie cavo, mientras los hombres de $4^{\circ}$ grado arrojaron el mayor tipo de pie normal.
Asimismo, otra investigación cuyos resultados fueron similares al presente proyecto son los obtenidos de la investigación denominada "la incidencia del pie plano y cavo en escolares" a cargo de Zurita (2009) realizado por el área de corporal de la Facultad de Ciencias de la Educación en la Universidad de Granada, España, que muestra algunas coincidencias con este estudio, donde a diferencia, se empleó la metodología de la huella plantar medida a través de un podógrafo para la recogida y toma de datos; esta referencia difiere del estudio actual que utilizo el "método Herzco". En cuanto a los resultados y conclusiones, se obtuvo que la incidencia de la patología plantar era de un 34,95\% (24,7\% de pie cavo y $10,25 \%$ de pie plano), muy similar a lo hallado en esta investigación, donde la mayor prevalencia fue el pie cavo; en cuanto a la edad, ellos hallaron valores incrementados en pies cavos en femeninas (elevación de la prevalencia coincidiendo con el inicio del desarrollo), mientras que en el pie plano, los valores son homogéneos a todas las edades; en la zona rural presentó índice más elevados de pie plano y normal, sin embargo, se obtuvieron valores muy bajos en patologías de pie cavo, por lo que representó a la única área que obtuvo valores más altos de pie plano que pie cavo, en el resto de las zonas, los valores son similares.

\section{Conclusiones}

En cuanto a las variables de la práctica deportiva, en la escuela deportiva, se encontró una antigüedad promedio de 23 meses, con una frecuencia de entrenamiento de dos días por semana. La posición de juego más común en los niños fue volante. El terreno de juego más empleado para la práctica de fútbol fue cancha de arena. El tipo de calzado más utilizado por los niños es el guayos tache redondo y el $6 \%$ usan regularmente plantillas.

Se encontró una prevalencia del dolor podal en el último año de $15.9 \%$ con una duración promedio de 2 días. La causa más común fue traumática, presentándose el dolor principalmente después (antes, durante, después) de la práctica deportiva. La localización predominante fue 
Kelly Díaz Theran - Podometría, práctica deportiva e índice de masa corporal asociada al dolor podal en niños

el retropié, y principalmente en el pie derecho (derecho, izquierdo, bilateral).

En cuanto a la asociación del dolor podal con las variables podométricas, de la práctica deportiva e Índice de Masa Corporal en niños de 12 a 14 años de escuelas deportivas de fútbol de la ciudad de Sincelejo. No se encontraron diferencia significativa entre estas variables entre los grupos de niños evaluados.

\section{Referencias bibliográficas}

Arizmendi, A., Pastrana, E., \& Rodriguez, B. (2004). Prevalencia de pie plano en niños de Morelia. Revista mexicana de Pediatría, 71(2), 66-69.

Aydog, S., Özçakar, L., Tetik, O., Demirel, H., \& Doral, M. (2005). Relation between foot arch index and ankle strength in elite gymnasts: a preliminary study. British Journal of Sports Medicine, 39(13).

Berdejo, D., Lara, A., Martínez, E., Cachón, J., \& Lara, S. (2013). Alteraciones de la huella plantar en función de la actividad física realizada. Revista Internacional de Medicina y Ciencias de la Actividad Física y el Deporte, 1(49), 19-39.

Cantó, L., García, J., Cantó, F., \& Padrós, F. (2007). Las Botas de fútbol un calzado deportivo especial. Congreso Nacional de Pedología. Bilbao, España.

Dvorak, J., Junge,L., Chomiak, J., \& Graf, T. (2000). Risk factor analysis for injuries in football players: Possibilities for a prevention program. Sports Med, 28(5), 69-74.

Elvira, J., Vera, F., Meana M. \& García J. (2008). Análisis biomecánico del apoyo plantar en la marcha atlética. Relación entre la huella plantar, ángulos de la articulación subastragalina y presiones plantares. European Journal of Human Movement, 20, 41-60.
Gómez, L., Franco, J., Nathy, J., Valencia, E., Vargas, D., \& Jiménez, L. (2009). Influencia del deporte en las características antropométricas de la huella plantar femenina. Revista Educación física y deporte, 28(2), 25-33.

Hamill, J., Bates, B., Knutzen, K., \& Kirpatrick G. (1989). Relationship between selected static and dynamic lower extremity measures. Clinical Biomechanics, 4, 217-225.

Hernández. R. (2006) Prevalencia del pie plano en niños y niñas en las edades de 9 a 12 años. Revista Internacional de Medicina y Ciencias de la Actividad Física y el Deporte, 6(23), 165-172.

Hernandez, R. (1999). Morfología Funcional Deportiva. Sistema Locomotor, 80.

López, et al. (2006). Respuestas, adaptaciones y simetría de la huella plantar producidas por la práctica de la marcha atlética. Cultura, ciencia y deporte, 3(2), 21-26.

Sirgo, G., \& Aguado, X. (1991). Estudio del comportamiento de la huella plantar en jugadores de voleibol después del esfuerzo considerando su composición corporal y somatotipo. Apuntes Medicina del deporte, 18, 207-212.

Wilson, A., Samuelson, B., \& Palermo, T. (2010). Obesity in children and adolescents with chronic pain: associations with pain and activity limitations. Clin J Pain, 26(8), 705711.

Zurita, A. (2009). Incidencia del pie plano y cavo en escolares. Recuperado de http://www.imbiomed.com.mx/1/1/ articulos. php?method=showDetail\&id_ articulo $=69072 \&$ id_seccion $=2176 \&$ id ejemplar=6912\&id_revista $=123$ 